\title{
Prevalencia de Eimeria sp. en bovinos de la cuenca ganadera de Florida - Pomacochas, Región Amazonas
}

\section{Prevalence of Eimeria sp. in bovines of the cattle basin of Florida - Pomacochas, Region Amazonas}

\section{RESUMEN}

\author{
${ }^{a}$ Joseph F. Bardales ${ }^{1},{ }^{b}$ William Bardales ${ }^{1},{ }^{c}$ Yander M. Briceño ${ }^{1}$ y Jhony A. Gonzales ${ }^{1 *}$
}

Los exámenes coproparasitológicos cualitativos y cuantitativos en bovinos se realizaron con el fin de determinar la prevalencia de Eimeria sp. en bovinos de la cuenca ganadera de Florida - Pomacochas, Región Amazonas. Se colectaron 103 y 29 muestras fecales de bovinos hembras y machos, respectivamente, durante los meses de octubre a diciembre del 2016. Los resultados obtenidos mediante la técnica de sedimentación mostraron una prevalencia de Eimeria sp. de $76,4 \%$ y $23,6 \%$ para hembras y machos, respectivamente. Finalmente, la presencia del $61,3 \%$ de Eimeria sp. en vacas fue significativamente diferente en relación con las otras categorías.

Palabras Clave: Eimeria sp., sedimentación, prevalencia.

\begin{abstract}
Qualitative and quantitative coproparasitological tests were performed in bovine with the aim to determine the Eimeria sp. prevalence in livestock breeding areas of Florida-Pomacochas. 29 and 103 fecal samples were collected from male and female bovines from October to December 2016. Results were obtained through the sedimentation technique. A prevalence of $76,4 \%$ and $23,6 \%$ of Eimeria sp. were found, for female and male, respectively. And a prevalence of $61,3 \%$ for cows being significantly different than other categories.
\end{abstract}

Keywords: Eimeria sp., sedimentation, prevalence.

\footnotetext{
${ }^{1}$ Universidad Nacional Toribio Rodríguez de Mendoza de Amazonas (UNTRM-A), Laboratorio de Enfermedades Infecciosas y parasitarias de Animales Domésticos de la UNTRM-A, Facultad de Ingeniería Zootecnista, Agronegocios y Biotecnología, Calle Higos Urco N 342-350-356, Calle Universitaria $N^{\circ} 304$, Chachapoyas, Perú

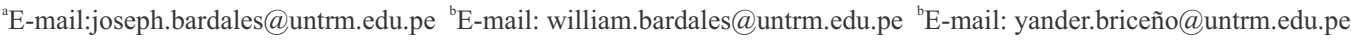
"Autor de correspondencia: E-mail: jhony.gozales@untrm.edu.pe
} 


\section{INTRODUCCIÓN}

La coccidiosis bovina es una enfermedad intestinal causada por protozoos del género Eimeria que afecta comúnmente a los animales jóvenes, infectándose estos por la ingestión de ooquistes esporulados que contaminan el pasto, el agua y el pienso, o al lamer pelaje contaminado (Jiménez, 2005).

Esta enfermedad está caracterizada clínicamente por diarrea sanguinolenta, anorexia, deshidratación, anemia, y muerte; la presentación subclínica de la enfermedad se caracteriza por una disminución en el consumo de alimento, baja conversión alimenticia y disminución en el ritmo de crecimiento del animal (Medina, 2007).

La cuenca ganadera de Florida - Pomacochas, Región Amazonas se encuentra a 2220 m.s.n.m., con una temperatura promedio anual de $15^{\circ} \mathrm{C}$ y una precipitación pluvial de 1104,2 mm anuales (Barboza et al., 2014), la cual hace propicio el desarrollo de esta enfermedad, que afecta la economía campesina básicamen- te solventada por la crianza de ganado vacuno.

La raza, el sexo y la categoría del animal pueden ser factores predisponentes a la suceptibilidad o resistencia de esta enfermedad; pero, en la Región Amazonas no existe información relacionada con este asunto; por esta razón la presente investigación tiene como objetivo determinar la prevalencia de Eimeria sp. en la cuenca ganadera de Florida - Pomacochas (Región Amazonas).

\section{MATERIALES Y MÉTODOS}

Área y animales estudiados

La investigación se realizó entre los meses de octubre y diciembre del 2016 en la cuenca ganadera de Florida - Pomacochas, Región Amazonas (Figura 1). Se analizaron 132 muestras fecales correspondientes al mismo número de vacunos de las razas Simmental y Brown Suiss, de ambos sexos y edades comprendidas entre cero y más de 36 meses.
PERÚ

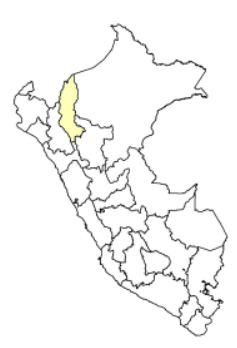

AMAZONAS

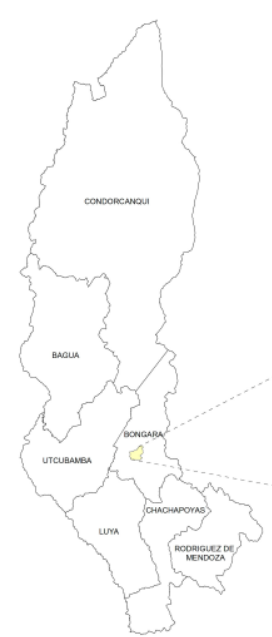

MICROCUENCA POMACOCHAS

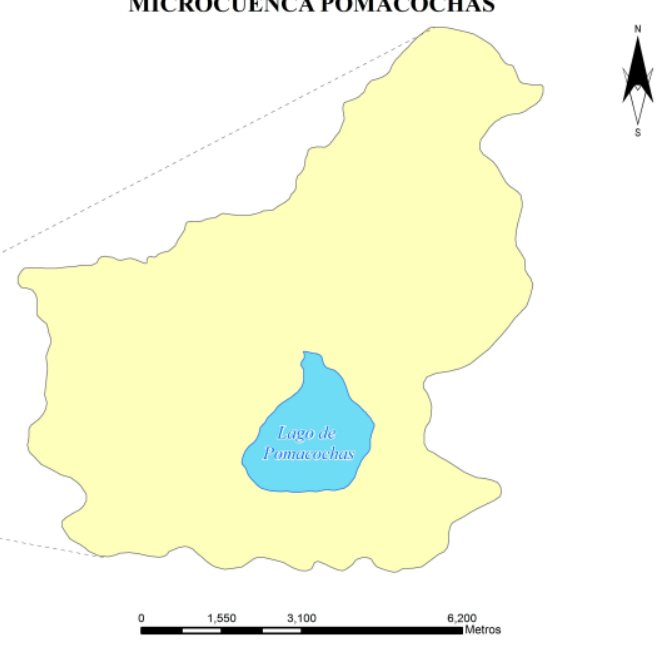

Figura 1. Ubicación de la cuenca ganadera de Florida-Pomacochas. 


\section{Análisis parasitológicos}

Las muestras fecales, de aproximadamente $20 \mathrm{~g}$ por unidad, fueron colectadas en las primeras horas de la mañana por vía rectal en bolsas de polietileno debidamente identificadas y almacenadas en refrigeración para su transporte.

La evaluación coproparasitológica cualitativa y cuantitativa se realizó en el Laboratorio de Enfermedades Infecciosas y Parasitarias de la Universidad Nacional Toribio Rodríguez de Mendoza de Amazonas (UNTRM-A) mediante las técnicas de sedimentación (Urquhart, 2001) y Mc Master (Rojas, 2004), respectivamente.

\section{Análisis estadístico}

Se calculó la prevalencia de Eimeria sp. mediante la determinación del número de muestras fecales positivas con un intervalo de confianza del 95\%. Las variables "procedencia", "sexo", "categoría" y "raza" se analizaron con el uso del software estadístico SPSS versión 20.0, utilizando estadística descriptiva, tablas de contingencia y la prueba Chi cuadrado.

\section{RESULTADOS}

Prevalencia de Eimeria sp. en la cuenca ganadera de Florida-Pomacochas

La tabla 1 muestra que de los 132 animales muestreados 106 presentaron Eimeria sp., y sólo en 26 animales no se observaron formas parasitarias. Estos datos representan el 80,3\% y 19,7\%, para presencia y ausencia de este parásito, respectivamente.
Tabla 1. Prevalencia de Eimeria sp. en Florida-Pomacochas

\begin{tabular}{lcc}
\hline \multicolumn{1}{c}{ Parásito } & $\mathbf{N}^{\circ}$ de animales & Porcentaje \\
\hline Eimeria sp. & 106 & $80,3 \%$ \\
NOFP & 26 & $19,7 \%$ \\
Total & 132 & $100,0 \%$ \\
\hline
\end{tabular}

NOFP: No se Observaron Formas Parasitarias

\section{Prevalencia de Eimeria sp. en bovinos según sexo}

La tabla 2, muestra que a partir del $80,3 \%$ de los animales que presentaron la enfermedad, el 76,4\% fueron hembras, y sólo el 23,6\% fueron machos. Sin embargo, no se observaron diferencias significativas en la prevalencia de Eimeria sp. según sexo debido a la alta diferencia entre el número de animales.

Tabla 2. Prevalencia de Eimeria sp. según sexo

\begin{tabular}{|c|c|c|c|c|}
\hline \multirow{2}{*}{ Parásitos } & \multirow{2}{*}{ Variable } & \multicolumn{2}{|c|}{ Sexo } & \multirow{2}{*}{ Total } \\
\hline & & Mach & Hembra & \\
\hline \multirow{2}{*}{ Eime } & $\mathrm{N}^{\circ}$ bov & 25 & 81 & 106 \\
\hline & $\%$ de & $23,6 \%$ & $76,4 \%$ & $100,0 \%$ \\
\hline \multirow{2}{*}{ NOFP } & $\mathrm{N}^{\circ}$ bo & 4 & 22 & 26 \\
\hline & $\%$ de sexo & $15,4 \%$ & $84,6 \%$ & $100,0^{\circ}$ \\
\hline
\end{tabular}

NOFP: No se Observaron Formas Parasitarias

Prevalencia de Eimeria sp. en bovinos según categoría Los resultados señalan que existen diferencias significativas en la prevalencia de Eimeria sp. según categoría siendo mayor en vacas $(61,3 \%)$, seguido por terneros y vaquillonas, con $9,9 \%$ y $9,4 \%$, respectivamente. Estos resultados podrían estar influenciados por el alto número de animales en esta categoría (Tabla 3 ).

Tabla 3. Prevalencia de Eimeria sp. en bovinos según categoría

\begin{tabular}{|c|c|c|c|c|c|c|c|}
\hline \multirow{2}{*}{ Parásitos } & \multirow{2}{*}{ Variables } & \multicolumn{5}{|c|}{ Categoría } & \multirow{2}{*}{ Total } \\
\hline & & Toro & Vaca & Torete & Vaquillona & Ternero & \\
\hline \multirow{3}{*}{ Eimeria sp. } & $\mathrm{N}^{\circ}$ de bovinos & 9 & 65 & 1 & 10 & 10 & 106 \\
\hline & $\%$ del parásito & $8,5 \%$ & $61,3 \%$ & $0,9 \%$ & $9,4 \%$ & $9,9 \%$ & $100,0 \%$ \\
\hline & $\%$ dentro de la categoría & $90,0 \%$ & $77,4 \%$ & $33,3 \%$ & $76,9 \%$ & $96,2 \%$ & $80,3 \%$ \\
\hline \multirow{3}{*}{ NOFP } & $\mathrm{N}^{\circ}$ de bovinos & 1 & 19 & 2 & 3 & 1 & 26 \\
\hline & $\%$ del parásito & $3,8 \%$ & $73,1 \%$ & $7,7 \%$ & $11,5 \%$ & $1,9 \%$ & $100,0 \%$ \\
\hline & $\%$ dentro de la categoría & $10,0 \%$ & $22,6 \%$ & $66,7 \%$ & $23,1 \%$ & $3,8 \%$ & $19,7 \%$ \\
\hline \multirow{3}{*}{ Total } & $\mathrm{N}^{\circ}$ de bovinos & 10 & 84 & 3 & 13 & 22 & 132 \\
\hline & $\%$ del parásito & $7,6 \%$ & $63,6 \%$ & $2,3 \%$ & $9,8 \%$ & $8,3 \%$ & $100,0 \%$ \\
\hline & $\%$ dentro de la categoría & $100,0 \%$ & $100,0 \%$ & $100,0 \%$ & $100,0 \%$ & $100,0 \%$ & $100,0 \%$ \\
\hline
\end{tabular}

NOFP: No se Observaron Formas Parasitarias 


\section{Prevalencia de Eimeria sp. en bovinos según raza}

La Tabla 4 se muestra que la razas Simmental y Brown Swiss presentaron prevalencia de Eimeria sp. con $54,7 \%$ y $45,3 \%$ de prevalencia respectivamente, pero sin existencia de diferencias significativas entre ambas.

Tabla 4. Prevalencia de Eimeria sp. en bovinos según raza

\begin{tabular}{ccccc}
\hline \multirow{2}{*}{ Parásito } & Variable & \multicolumn{2}{c}{ Raza } & \multirow{2}{*}{ Trown } \\
\cline { 3 - 4 } & & Swiss & Simmental & \\
\hline \multirow{2}{*}{ Eimeria } & $\mathrm{N}^{\circ}$ bovinos & 48 & 58 & 106 \\
sp. & $\%$ del parásito & $45,3 \%$ & $54,7 \%$ & $100,0 \%$ \\
& $\%$ dentro raza & $77,4 \%$ & $82,9 \%$ & $80,3 \%$ \\
\multirow{2}{*}{ NOFP } & $\mathrm{N}^{\circ}$ bovinos & 14 & 12 & 26 \\
& $\%$ del parásito & $53,8 \%$ & $46,2 \%$ & $100,0 \%$ \\
& $\%$ dentro raza & $22,6 \%$ & $17,1 \%$ & $19,7 \%$ \\
Total & $\%$ de bovinos & 62 & 70 & 132,0 \\
& $\%$ del parásito & $47,0 \%$ & $53,0 \%$ & $100,0 \%$ \\
& $\%$ dentro raza & $100,0 \%$ & $100,0 \%$ & $100,0 \%$ \\
\hline
\end{tabular}

NOFP: No se Observaron Formas Parasitarias

\section{DISCUSIÓN}

Los resultado obtenidos muestran una prevalencia total del 80,3\% de Eimeria sp. para todos los animales muestreados, siendo mayor en hembras con $76,4 \%$, y sólo $23,6 \%$ en machos. Estos resultados son similares a los encontrados por Colina et al. (2013), dónde encontraron una prevalencia del $84,3 \%$ en hembras, y del $87,1 \%$ en machos, sin diferencias significativas entre ambos sexos, como ocurre en el presente estudio. Sobre la variable categoría, los resultados muestran mayor prevalencia de Eimeria sp. en vacas con el $61,3 \%$, seguido por terneros y vaquillonas con un $9,9 \%$ y $9,4 \%$, respectivamente. A diferencia de lo que ocurre con la variable anteriormente citada, estos resultados son diferentes a los encontrados por Díaz et al. (1998), quienes observaron tasas de positividad para esta enfermedad del $65 \%$ para bovinos de 2 a 6 meses de edad y lo relacionaron con el contacto de los terneros con animales adultos portadores de la enfermedad.

La prevalencia de Eimeria sp. según sexo no mostró diferencias significativas entre estos dos factores, obteniendo un porcentaje de 54,7\% y 45,3\% en bovinos de la raza Simmental y Brown Swiss, respectivamente. Estos resultados podrían estar relacionados con lo expuesto por Suárez et al. (1995), quienes indican que los bovinos Bos Taurus poseen genes menos resistentes a infecciones de parásitos gastrointestinales en comparación con bovinos Bos indicus.

Los resultados obtenidos en están investigación estarían relacionados con las condiciones tropicales húmedas de la cuenca ganadera de Florida - Pomacochas, aumentando la contaminación fecal de los alimentos y por tanto presentándose brotes agudos de enfermedades derivadas. El conocimiento de la prevalencia de la coccidiosis con los datos disponibles es variable de acuerdo a la región y época del año, pero son necesarios para ser considerados como parte de un programa de salud animal (Quiroz, 2005).

\section{CONCLUSIÓN}

Se determinó la prevalencia de Eimeria sp. en la cuenca ganadera de Florida - Pomacochas según el sexo, categoría y raza, mostrando mayor prevalencia en vacas en comparación con otras categorías. Asimismo se determinó que las variables sexo y raza no tienen un efecto significativo en la presencia de éste parásito.

\section{REFERENCIAS BIBLIOGRÁFICAS}

Barboza, E., J.L. Maicelo, C.N.V. Mestanza, J. Castro, y S.M. Oliva "Análisis morfométrico y batimétrico del lago Pomacochas (Perú)". INDES Revista de Investigación para el Desarrollo Sustentable, 2(2014): 90-97.

Colina, J.C., G.A. Mendoza, y C.A. Jara. "Prevalencia del parasitismo por Eimeria en bovinos, Bos taurus, del Distrito Pacanga ( La Libertad, Perú ) y su relación con factores sociodemográficos y ambientales". Rebiolest, 1(2013): 72-78.

Díaz, A., J. Justo, M. Gonzáles, E. Piña, y L. Ramírez. "Prevalencia de coccidiosis en bovinos de los llanos de Monay, estado Trujillo, Venezuela”, Revistacientifica, 67, (1998): 46-53.

Jiménez, A. "Coccidiosis Bovina". CySB, 17(2005): 48-53.

Medina, M. “Coccidiosis”. México, 2007. Recupera- 
do de: http://www.ammveb.net/clinica/ coccidiosis.pdf

Quiroz, H. "Parasitología y enfermedades parasitarias de animales domésticos". México, 2005. Recuperado de: https://books.google.com. pe/books?id=xRxkXaI1Y6EC\&pg=PA128\& lpg $=$ PA128\&dq $=$ susceptibilidad + de + ternero $\mathrm{s}+\mathrm{a}+$ enfermedades + parasitarias $\&$ source $=\mathrm{bl}$ \&ots $=k \_n W c p-y l I \& s i g=d g 4 n l V F E j \_r h$ E B u ZW 0 D fIB 0 - rf A \& h $1=$ es \& sa $=X$ $\& v e d=0$ ahUKEwjy6ryln8_UAhVBeCYKH XD_B10Q6AEIMTAC\# $\mathrm{v}=$ onepage $\& \mathrm{q}=$ susc eptibilidad de terneros a enfermedades parasitarias $\& \mathrm{f}=$ false

Rojas, M. "Nosoparasitosis de los Rumiantes Domésticos Peruanos". Lima (Perú), 2004.

Suárez, V., M. Busetti, y F. Babinec. "Comparative effects of nematode infection on Bos taurus and Bos indicus crossbred calves grazing on Argentina's western pampas". Vet. Parasitol., 53(1995): 263-271.

Urquhart, G.M. "Parasitología Veterinaria”. Zaragoza (España), 2001. 